\title{
Propuesta de Clasificación de Huesos Suturales
}

\author{
Classification Proposal of Sutural Bones
}

Juan Silva $^{1,2}$; Claudia Araya ${ }^{1,3}$; Samara Liberona ${ }^{4}$; Pablo Otárola ${ }^{1}$ \& Rodrigo Donoso ${ }^{1}$

SILVA, J.; ARAYA, C. ; LIBERONA, S.; OTÁROLA, P. \& DONOSO, R. Propuesta de clasificación de huesos suturales. Int. J. Morphol., 39(3):766-772, 2021.

RESUMEN: Los huesos suturales tienen importancia anatómica y médico-legal. Se observan en las suturas de la cabeza ósea y tradicionalmente son descritos como planos, supernumerarios, irregulares, inconstantes, independientes y de variable morfología y frecuencia. Actualmente, no existe una clasificación única de estos huesos que incorpore todas las categorías descritas en la literatura. El objetivo de este trabajo fue proponer una clasificación actualizada de estos elementos, en función de una revisión bibliográfica exhaustiva y el análisis de cráneos de individuos chilenos. La muestra utilizada correspondió a 113 huesos suturales presentes en 12 cráneos secos, de individuos adultos chilenos. En la clasificación se consideró su ubicación en la cabeza (cráneo o cara), su origen embrionario, su relación con una fontanela, su forma, su posición en la cara y su relación con las tablas óseas. Los datos obtenidos se registraron en formulario especialmente diseñado y se tomaron fotografías digitales. Como resultado general, pudimos desarrollar una propuesta de clasificación de huesos suturales completa y satisfactoria y con ella se analizó los huesos de la muestra, pudiendo evidenciar la presencia de todas las categorías del instrumento. También pudimos constatar que en la totalidad de los cráneos utilizados se observaron huesos suturales, que los cráneos masculinos presentan un mayor número de estos huesos, pero que en los cráneos femeninos fue posible reconocer todas los tipos de huesos suturales, entre otros resultados. También se evidenció un tipo de hueso sutural no descrito anteriormente, el hueso sutural craneal puntiforme. Como conclusión de este trabajo, es importante destacar que los huesos suturales presentan características comunes a otras estructuras utilizadas en identificación forense, es decir, son perennes, únicos, de fácil observación, de fácil comparación y gran variabilidad, por estas razones la presente propuesta de clasificación permite ser planteada como una metodología auxiliar en la identificación humana.

PALABRAS CLAVE: Osteología; Cráneo; Huesos suturales; Huesos supernumerarios; Identificación forense.

\section{INTRODUCCIÓN}

Los huesos suturales (HS) corresponden a huesos planos, supernumerarios, irregulares, inconstantes e independientes. Se observan, entre las suturas y fontanelas de la cabeza ósea (cráneo y cara), siendo huesos de muy variable morfología y frecuencia. Están formados desde centros de osificación normales o adicionales, relacionados con variables étnicas y considerados de importancia genética y hereditaria (Cruveilhier, 1851; Testut et al., 1965; Aprile et al., 1971; Lockhart et al., 1988; Williams et al., 1998; GarcíaHernández et al., 2007; García-Hernández \& Murphy, 2009; Latarjet \& Ruiz-Liard, 2010; Silva \& Araya, 2016). La clasificación de estos huesos ha sido bastante compleja y poco satisfactoria, debido a sus particulares características y gran variabilidad.
Uno de los primeros anatomistas que los describió fue Worm (Danés 1588 - 1654, médico), quién en 1741 escribe a Thomas Bartholin (Danés 1616 - 1680, médico) detallando estos pequeños huesos hallados en el cráneo (Romero, 2017). Además, Worm indica que Hipócrates fue el primero en mencionarlos y describirlos como huesos triangulares en la sutura lambdoidea y en el proceso mastoides.

Según Rambaud \& Renault (1864), el origen embrionario de estos huesos comenzaría al quinto mes de vida fetal. Desde la línea sagital del cráneo, en la parte superior de la escama y fontanela posterior. Durante el octavo mes, algunos de estos puntos se sueldan formando una hoja triangular rodeada de cartílago.

\footnotetext{
${ }^{1}$ Facultad de Medicina y Ciencia, Universidad San Sebastián, Chile.

${ }^{2}$ Centro de Investigación en Educación, Universidad Bernardo O’Higgins, Chile.

${ }^{3}$ Programa de Especialidad de Ortodoncia y Ortopedia Dento-Maxilo-Facial, Escuela de Graduados, Facultad de Odontología, Universidad de Chile, Chile.

${ }^{4}$ Facultad de Ciencias para el Cuidado de la Salud, Universidad San Sebastian, Chile.
} 
La clasificación descrita en la literatura incluye actualmente, a los HS verdaderos (de origen membranoso y provenientes de un centro de osificación adicional) y HS falsos (de origen cartilaginoso y provenientes de un centro de osificación normal y que ha permanecido independiente) (Testut et al.; Rouvière \& Delmas, 1999; Latarjet \& RuizLiard). También se han clasificado según la posición que ocupan en la cabeza ósea (fontanelares y suturales) (Poirier et al., 1906; Testut et al.; Orts Llorca, 1959; Latarjet \& RuizLiard). Testut et al. describen HS observables en las caras exocraneal y endocraneal de la cabeza ósea. Parker (1905) reconoce y agrega a esta clasificación los HS presentes en la órbita y finalmente, Silva \& Araya incorporan los HS umbilicados.

La información sobre estos huesos no solo tiene importancia anatómica, sino que también puede tener gran relevanciaen la identificación médico-legal, ya que son estructuras anatómicas perennes, únicas, de fácil observación, fácil comparación y gran variabilidad, lo que permite desarrollar un método de identificación humana de bajo costo, que requiere de solo una imagen de comparación (Rx, TAC o RMN) y personal capacitado.
El objetivo de este trabajo fue proponer una clasificación actualizada de los HS, en función de una revisión bibliográfica exhaustiva y el análisis de cráneos de individuos chilenos.

\section{MATERIAL Y METODO}

La muestra correspondió a 113 HS observados en 12 cráneos secos, no descalotados, en buen estado de conservación, de individuos adultos chilenos, de ambos sexos (6 masculinos y 6 femeninos). Este material óseo pertenece a la osteoteca de la Facultad de Medicina y Ciencia de la Universidad San Sebastián. El trabajo de observación realizado en estos cráneos, no significó la destrucción de este valioso material.

Para la confección de la Propuesta de Clasificación de Huesos Suturales (PCHS) observable en la Tabla I, hemos considerado su ubicación general en la cabeza ósea, es decir cráneo o cara (así pudimos diferenciar a los HS craneales de los faciales), su origen embrionario (esto nos

Tabla I. Propuesta de clasificación de huesos suturales.

\begin{tabular}{|c|c|c|c|c|c|}
\hline \multicolumn{6}{|c|}{ PROPUESTA DE CLASIFICACION DE HUESOS SUTURALES } \\
\hline \multirow{7}{*}{ 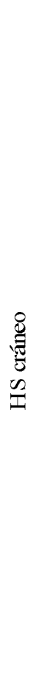 } & \multirow{3}{*}{ 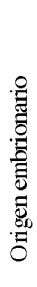 } & & Clasificación HS & Abreviatura & Descripción del hueso sutural \\
\hline & & 1 & Hueso sutural craneal verdadero & HSCV & $\begin{array}{l}\text { Hueso supernumerario del cráneo, ubicado en una sutura craneal, su o rigen embrionario no } \\
\text { corresponde a un punto de osificación normal de uno de los huesos del cráneo. Por lo tanto, } \\
\text { proviene de un punto de osificación extra (supernumerario verdadero). }\end{array}$ \\
\hline & & 2 & Hueso sutural craneal falso & HSCF & $\begin{array}{l}\text { Hueso supernumerario del cráneo, ubicado en una sutura craneal, su origen embrionario } \\
\text { corresponde a un punto de osificación normal de alguno de los huesos del cráneo, pero que ha } \\
\text { quedado independiente durante su desarrollo. }\end{array}$ \\
\hline & \multirow{2}{*}{ 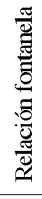 } & 3 & Hueso sutural craneal fontanelar & HSCFO & Hueso supernumerario del cráneo, ubicado en una fontanela. \\
\hline & & 4 & Hueso sutural craneal no fontanelar & HSCNFO & $\begin{array}{l}\text { Hueso supernumerario del cráneo, ubicado en una sutura craneal, pero alejado de alguna de las } \\
\text { fontanelas. }\end{array}$ \\
\hline & \multirow{2}{*}{ 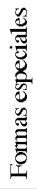 } & 5 & Hueso sutural craneal umbilicado & $\mathrm{HSCU}$ & Hueso supernumerario del cráneo, que en su estructura presenta un ombligo óseo. \\
\hline & & 6 & Hueso sutural craneal puntiforme & HSCP & $\begin{array}{l}\text { Hueso supernumerario del cráneo, ubicado en cercanía de una sutura craneal, de tamaño pequeño } \\
\text { y de forma circular que asemeja un punto y no es parte de un hueso sutural craneal umbilicado. }\end{array}$ \\
\hline \multirow{5}{*}{ 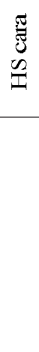 } & \multirow{2}{*}{$\frac{\sqrt[3]{8}}{8}$} & 7 & Hueso sutural facial orbitario & HSFO & Hueso supernumerario de la cara, ubicado en alguna de las paredes de la órbita. \\
\hline & & 8 & Hueso sutural facial lagrimal & HSFL & Hueso supernumerario de la cara, ubicado en alguna de las suturas alrededor del hueso lagrimal. \\
\hline & \multirow{3}{*}{ 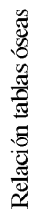 } & 9 & Hueso sutural exocraneal & HSEX & Hueso sutural craneal o facial observable solo en la tabla ósea externa. \\
\hline & & 10 & Hueso sutural endocraneal & HSEN & Hueso sutural craneal o facial observable solo en la tabla ósea interna. \\
\hline & & 11 & Hueso sutural de espesor total & HSET & $\begin{array}{l}\text { Hueso sutural craneal o facial observable en ambas tablas, atravesando de una hacia la otra y que } \\
\text { por tanto presenta el mismo grosor que los huesos vecinos. }\end{array}$ \\
\hline
\end{tabular}


permitió elaborar los ítems 1 y 2 de la clasificación), su relación con una fontanela (nos permitió elaborar los ítems 3 y 4), su forma (nos permitió establecer los ítems 5 y 6), su posición en la cara ósea (esta característica nos permitió diferenciar a los ítems 7 y 8) y finalmente si se observa en tabla ósea externa, interna o en ambas (esta última característica determino los ítems 9,10 y 11 ).

Cada uno de los huesos de la muestra fue analizado sistemáticamente e identificado según esta PCHS. Para el correcto registro de estos huesos presentes en los cráneos de la muestra, se fotografiaron con cámara digital marca $\mathrm{Ca}$ non $($ ) modelo PowerShot $\subset$ D $\mathrm{D} 30$. Todos los datos se registraron en formulario ad hoc.

\section{RESULTADOS}

El análisis y síntesis de la información disponible en la literatura anatómica nos permitió establecer una propuesta satisfactoria de clasificación de los HS. Esta propuesta y la descripción de sus elementos se puede observar en la Tabla I.

Es importante destacar que esta clasificación nos permitió incluir, por lo menos en una de sus opciones, a todos los HS evidenciados en la muestra, lo que permitió evidenciar que esta clasificación es satisfactoria. En las Figuras 1 y 2 se observan fotografías de HS representativos de cada categoría de la PCHS.
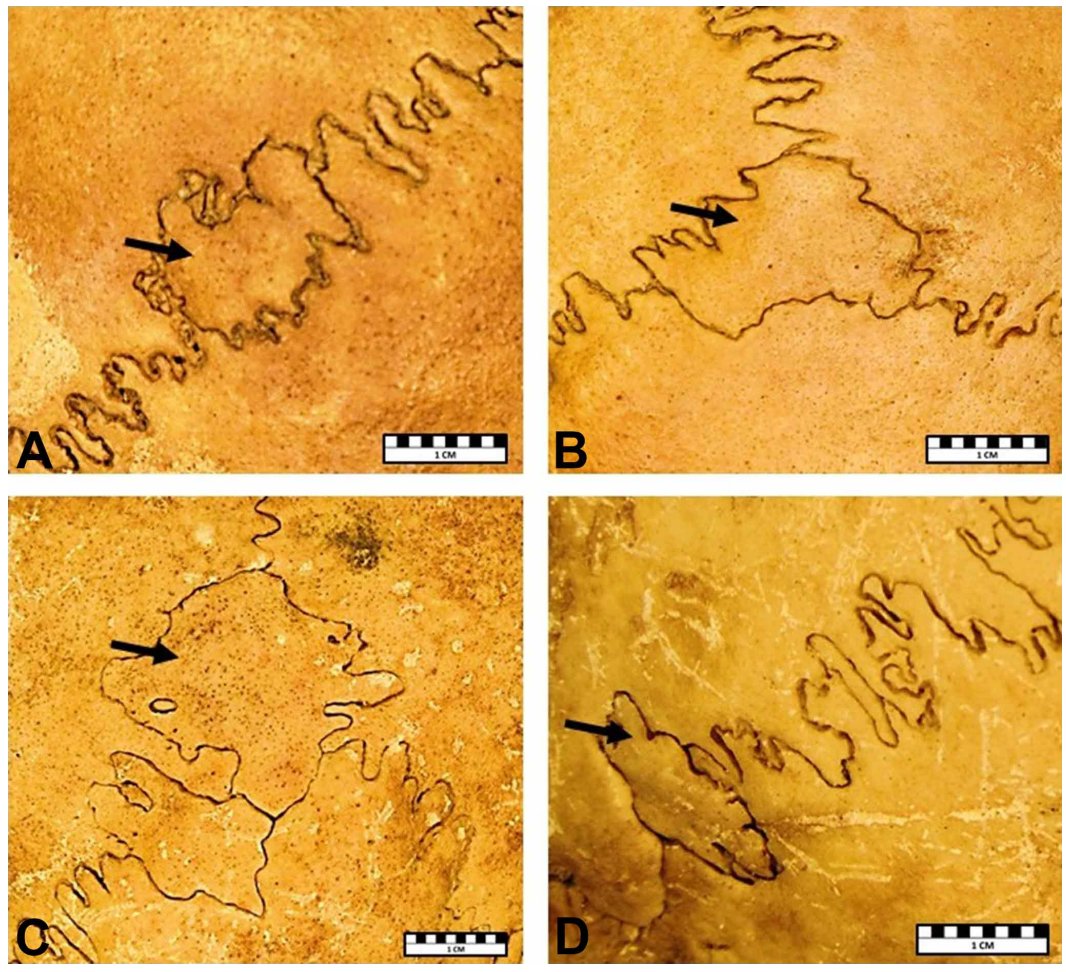

También es relevante indicar que no todos los ítems de la clasificación son excluyentes entre sí. Así las dos primeras categorías (HSCV y HSCF) permiten ser al mismo tiempo, catalogadas bajo otro ítem. Por ejemplo, un HS que ha sido clasificado como Hueso Sutural Craneal Falso, puede también ser clasificado como Hueso Sutural Craneal Fontanelar, complementando su clasificación. No ocurre lo mismo con aquellas clasificaciones pertenecientes a una dicotomía. Desde este último punto de vista, un Hueso Sutural Craneal Umbilicado no puede ser clasificado al mismo tiempo, como Hueso Sutural Facial Lagrimal.

En la Tabla II se identifican los distintos tipos de HS y las cantidades observadas en cada uno de los cráneos utilizados en este estudio. Se consideraron las variables de sexo, edad y clasificación de HS. El análisis de estos datos permitió determinar la presencia de estos huesos en el $100 \%$ de los cráneos, tanto en individuos de sexo masculino como femenino. Todos los HS observados pertenecen a la clasificación HSEX, y debido a que los cráneos no se descalotaron, no fue posible determinar si se presentan HSEN o HSET en el material óseo utilizado.

La distribución de los distintos HS dentro de la muestra sin diferenciar por sexo y utilizando las categorías de la PCHS se registran en la Figura 3 y su representación porcentual se observa en la Figura 4.

La distribución de los distintos HS dentro de la muestra diferenciadas por sexo y utilizando las categorías de la
Fig. 1. En A. fotografía de HSCV ubicado en sutura parieto-occipital izquierda de cráneo de individuo masculino (flecha). En B. fotografía de HSCF ubicado en posición lambda en cráneo de individuo masculino (flecha). En C. fotografía de HSCFO ubicado en lambda en cráneo de individuo masculino (flecha). En D. fotografía de HSCNFO en sutura parieto-occipital izquierda de cráneo de individuo masculino (flecha). 
PCHS se registran en la Figura 5 y su representación porcentual se observa en la Figura 6.

Es importante destacar algunos resultados interesantes, por ejemplo, los HSCF $(41,7 \%$ de la muestra) y los HSCNFO (38\% de la muestra), son los HS más frecuentes
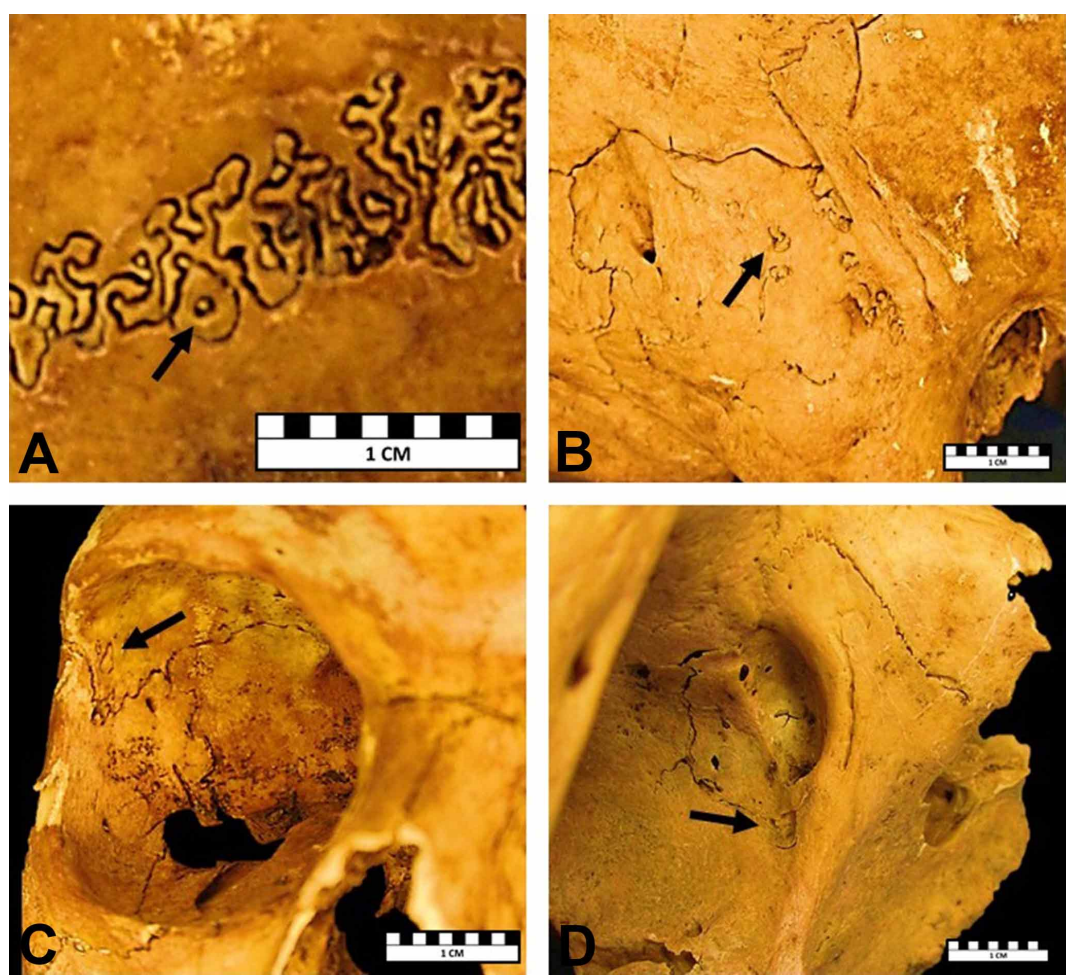

Fig. 2. En A. fotografía de HSCU ubicado en sutura fronto-parietal derecha en cráneo de individuo femenino (flecha). En B. fotografía de HSCP ubicado en escama de temporal derecho de individuo masculino (flecha). En C. fotografía de HSFO ubicado en pared lateral de orbita derecha en cara de individuo femenino (flecha). En D. fotografía de HSFL ubicado en pared inferior de órbita derecha en cara de individuo masculino (flecha).

Representación de los HS según la PCHS

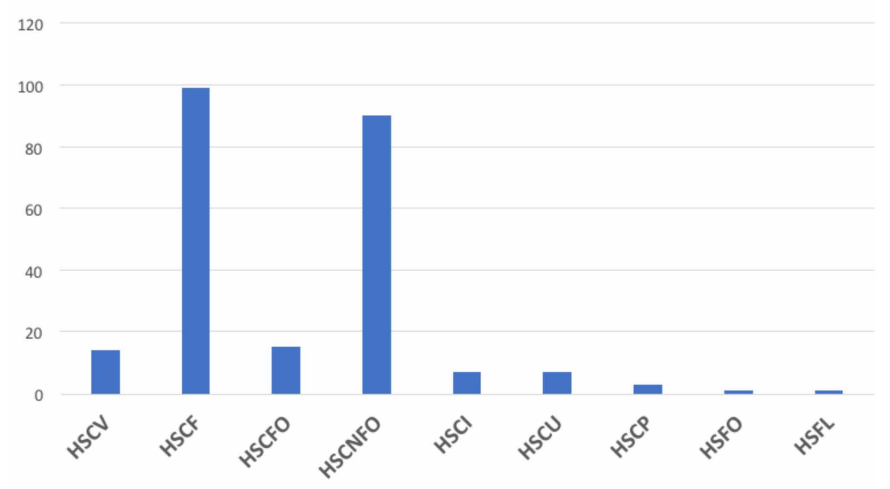

Fig. 3. Gráfico de barras que representa la distribución de los HS según PCHS de la muestra en estudio, sin diferenciar por sexo. sin diferenciar por sexo. Estos resultados se mantienen al diferenciar por sexo tanto en cráneos masculinos (HSCF: $40 \%$ / HSCNFO: $35 \%$ ) como en cráneos femeninos (HSCF: 44,3 \% / HSCNFO: 42,3 \%). Además, los cráneos masculinos presentan una mayor cantidad de HS (1,5 veces más que en los cráneos femeninos). Pero los cráneos femeninos presentan una mayor variabilidad, evidenciando la totalidad de categorías de la PCHS (ya que en cráneos masculinos no se hallaron HSCP, HSFO ni HSFL).

Como resultado inesperado del estudio, se logró identificar un tipo de HS no descrito en la literatura. Este hueso debido a sus características morfológicas, lo denominamos Hueso Sutural Craneal Puntiforme (HSCP) y fue inmediatamente incorporado a la PCHS, complementando dicha metodología (en la Figura $2 b$, es posible observar uno de ellos en proceso mastoides de hueso temporal derecho).

HS sin diferenciar por sexo

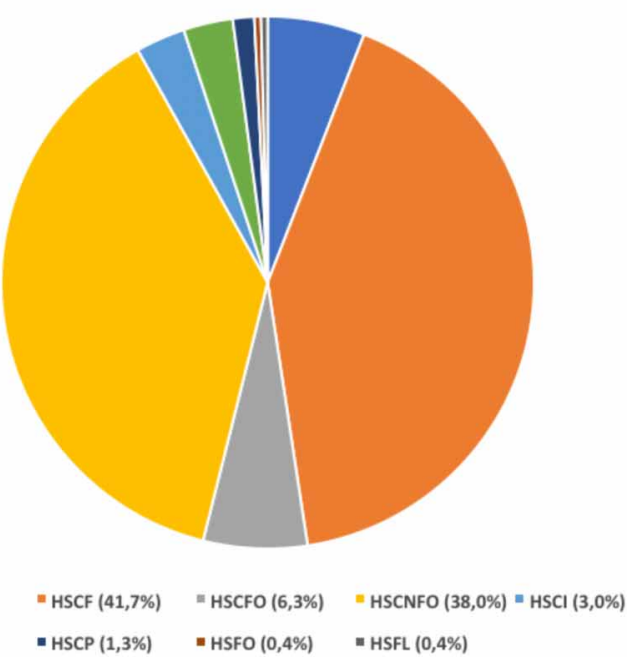

Fig. 4. Gráfico circular que representa la distribución porcentual de los HS según PCHS de la muestra en estudio, sin diferenciar por sexo. 


\section{Resultados diferenciados por sexo}

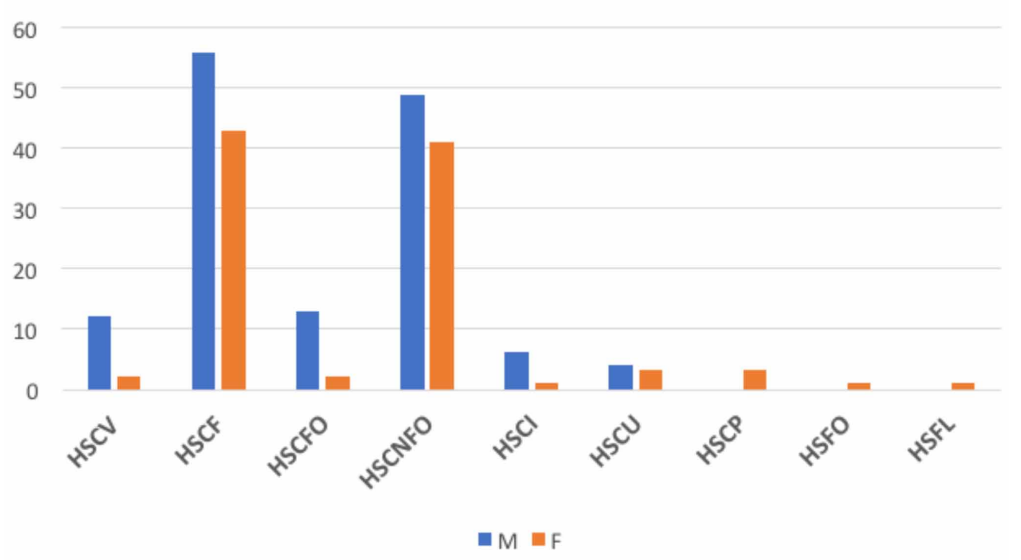

HS en cráneos masculinos

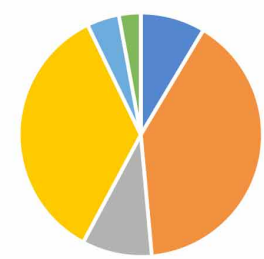

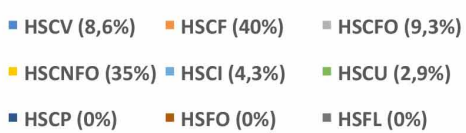

HS en cráneos femeninos

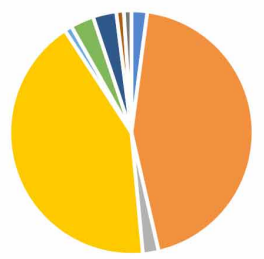

$$
\begin{aligned}
& \text { " } \operatorname{HSCV}(2,1 \%) \quad \text { " } \operatorname{HSCF}(44,3 \%) \quad \text { "HSCFO }(2,1 \%) \\
& \text { " } \operatorname{HSCNFO}(42,3 \%)=\operatorname{HSCl}(1 \%) \quad \| \operatorname{HSCU}(3,1 \%) \\
& \text { - } \operatorname{HSCP}(3,1 \%) \quad-\operatorname{HSFO}(1 \%) \quad \operatorname{HSFL}(1 \%)
\end{aligned}
$$

Fig. 5. Gráfico de barras que representa la distribución de los HS según PCHS de la muestra en estudios, diferenciados por sexo.

Tabla II. Tabla de resultados totales presentes en la muestra, ordenados por sexo, edad y utilizando las categorías de las PCHS. Además, se incluyen los resultados totales sin diferenciar por sexo.

\begin{tabular}{ccccccccccc}
\hline \multicolumn{10}{c}{ Masculinos } \\
\hline $\mathrm{N}^{\circ}$ & SEXO & EDAD & HSCV & HSCF & HSCFO & HSCNFO & HSCU & HSCP & HSFO & HSFL \\
\hline 1 & M & $30-40$ & 0 & 8 & 0 & 8 & 0 & 0 & 0 \\
2 & M & $30-40$ & 2 & 6 & 2 & 6 & 0 & 0 & 0 \\
3 & M & $40-50$ & 3 & 8 & 4 & 7 & 0 & 0 & 0 \\
4 & M & $40-50$ & 1 & 16 & 1 & 10 & 0 & 0 & 0 & 0 \\
5 & M & $40-50$ & 3 & 12 & 3 & 12 & 0 & 0 & 0 & 0 \\
6 & M & $40-50$ & 3 & 6 & 3 & 6 & 4 & 0 & 0 & 0 \\
& \multicolumn{2}{l}{ Total Masculinos } & 12 & 56 & 13 & 49 & 4 & 0 & 0 & 0
\end{tabular}

\begin{tabular}{ccccccccccc}
\hline \multicolumn{10}{c}{ Femeninos } \\
\hline $\mathrm{N}^{\circ}$ & SEXO & EDAD & HSCV & HSCF & HSCFO & HSCNFO & HSCU & HSCP & HSFO & HSFL \\
\hline 1 & $\mathrm{~F}$ & $20-30$ & 2 & 28 & 2 & 28 & 1 & 0 & 0 \\
2 & $\mathrm{~F}$ & $20-30$ & 0 & 2 & 0 & 2 & 2 & 0 & 0 & 0 \\
3 & $\mathrm{~F}$ & $20-30$ & 0 & 5 & 0 & 4 & 0 & 0 & 0 & 1 \\
4 & $\mathrm{~F}$ & $30-40$ & 0 & 2 & 0 & 2 & 0 & 0 & 0 & 0 \\
5 & $\mathrm{~F}$ & $40-50$ & 0 & 1 & 0 & 1 & 0 & 1 & 0 & 0 \\
6 & $\mathrm{~F}$ & $50-60$ & 0 & 5 & 0 & 4 & 0 & 2 & 1 \\
\multicolumn{2}{l}{ Total Femeninos } & 2 & 43 & 2 & 41 & 3 & 3 & 1 \\
\end{tabular}




\section{DISCUSIÓN}

Tomando en consideración la información proveniente de la literatura, este trabajo logra proponer una clasificación ordenada y completa de los huesos suturales.

Hemos considerado las diversas clasificaciones descritas y con ellas logramos desarrollar una propuesta satisfactoria que tiende a incorporar a aquellos autores que prestaron especial atención a estos huesos (Poirier et al.: Testut et al.; Parker; Rouviére \& Delmas; Orts Llorca; Latarjet \& Ruiz-Liard; Silva \& Araya).

Interesante fue el encontrar en la revisión bibliográfica la descripción de los huesos insulares descritos como un tipo de HS, y luego, durante el proceso de observación del material óseo, confirmar la presencia de estos elementos. La definición tradicional de los HS indica que estos son posibles de observar, en las suturas y fontanelas del cráneo (Strambio, 1854; Poirier et al.; Orts Llorca; Testut et al.; Aprile et al.; Prives et al., 1984; Latarjet \& Ruiz-Liard; Murlimanju et al., 2011), pero la definición de los huesos insulares indica que son huesos que se desarrollan lejos de las suturas y fontanelas, en medio de algunos huesos planos, por su cara endocraneal (Poirier et al.; Testut et al.; Rouviére \& Delmas; Latarjet \& Ruiz-Liard), esta disonancia entre las definiciones nos permite afirmar que los huesos insulares son otro tipo de huesos supernumerarios de la cabeza ósea y no deben estar presente dentro de la PCHS aquí descrita.

No es menos cierto que la particular variabilidad de forma, posición y frecuencia de los HS probablemente ha dificultado el desarrollo de una clasificación clara. Consideramos también que la importancia de estos huesos ha sido poco reconocida, posiblemente por no presentar cuadros de interés médico relacionados con ellos.

\section{CONCLUSIÓN}

Este trabajo propone una nueva clasificación que resume toda la información disponible hasta el momento, sistematizándola en una sola herramienta de fácil comprensión y de gran utilidad.

También es un hecho muy interesante de destacar, la reconsideración de la clasificación de los huesos insulares, reconocidos ahora como una estructura independiente de los HS. No menor es la importancia de la identificación de un HS no descrito en la literatura, los HSCP.
Al igual que otras estructuras del cuerpo humano, los HS son relativamente perennes (cambian muy poco en el tiempo), son únicos, de fácil observación, de fácil comparación y gran variabilidad, lo que determina su utilidad en la identificación médico-legal, sobre todo en individuos jóvenes donde el proceso de osificación de las suturas del cráneo aún no ha ocultado las articulaciones alrededor de estos huesos. Por estas razones la PCHS permite ser utilizada como una metodología auxiliar en la identificación forense ya que permite identificar, caracterizar y clasificar a los HS de un individuo. Por lo tanto, las características propias de estos huesos (número, forma, posición y morfología) permiten diferenciar un cráneo de otro y por lo tanto un individuo de otro, pudiendo esclarecer la identidad indubitada de restos de individuos de identidad dubitada.

SILVA, J.; ARAYA, C. ; LIBERONA, S.; OTÁROLA, P. \& DONOSO, R. Classification proposal of sutural bones. Int. J. Morphol, 39(3):766-772, 2021.

SUMMARY: The sutural bones have anatomical and medico-legal importance. They are observed in the sutures of the bony head and are traditionally described as flat, supernumerary, irregular, inconstant, independent and of variable morphology and frequency. Currently, there is no single classification of these bones that incorporates all the categories described in the literature. The objective of this work was to propose an updated classification of these elements, based on an exhaustive bibliographic review and the analysis of the skulls of Chilean individuals. The sample used corresponds to 113 sutural bones present in 12 dry skulls of Chilean adults. The classification considers its location on the head (skull or face), its embryonic origin, its relationship to a fontanelle, its shape, its position on the face, and its relationship to bone tables. The data obtained was recorded in a specially designed format and digital photographs were taken. As a general result, we were able to develop a complete and satisfactory classification of sutural bones proposal and with it, the bones of the sample were analyzed, showing the presence of all categories of the instrument. We were also able to verify that sutural bones were observed in all the skulls used, that male skulls present a greater number of these bones, but that in the female skulls it was possible to recognize all types of sutural bones, among other results. A type of sutural bone not previously described, the shaped point cranial sutural bone, was also evidenced. As a conclusion to this work, it is important to highlight that sutural bones present characteristics common to other structures used in forensic identification, that is, they are perennial, unique, easily observed, easily compared and great variability, for these reasons the present classification proposal allows it to be proposed as an auxiliary methodology in human identification.

KEY WORDS: Osteology; Skull; Sutural bones; Supernumerary bones; Forensic Identification. 


\section{REFERENCIAS BIBLIOGRAFICAS}

Aprile, H.; Figún, M. \& Garino, R. Anatomía Odontológica Orocervicofacial. $5^{\mathrm{a}}$ ed. Buenos Aires, El Ateneo, 1971.

Cruveilhier, J. Traité d'Anatomie Descriptive. $3^{\mathrm{a}}$ ed. Paris, Labé Éditeur, Libraire de la Faculté de Médecine, 1851.

García-Hernández, F. \& Murphy-Echeverría, G. Frecuency of wormian lambdoid bone in skulls with artificial deformation in Northern Chile. Int. J. Morphol., 27(3):933-8, 2009.

García-Hernández, F.; Díaz, B. J. L. \& Murphy, E. G. T. Incidence of sutural bones in a population of chango skulls of Northern Chile, Antofagasta. Int. J. Morphol., 25(4):801-4, 2007.

Latarjet, M. \& Ruiz-Liard, A. Anatomía Humana. $4^{\mathrm{a}}$ ed. Buenos Aires, Panamericana, 2010.

Lockhart, R.; Hamilton, G. \& Fyfe, F. Anatomía Humana. Ciudad de México, Interamericana, 1988

Murlimanju, B. V.; Prabhu, L. V.; Ashraf, C. M.; Kumar, C. G.; Rai, R. \& Maheshwari, C. Morphological and topographical study of Wormian bones in cadaver dry skulls. J. Morphol. Sci., 176-9, 2011.

Orts Llorca, F. Anatomía Humana. 2a ed. Valencia, Científico-Médica, 1959.

Parker, C. H. Wormian bones. Chicago, Robert Press, 1905.

Poirier, P.; Charpy, A. \& Cuneo, B. Tratado Elemental de Anatomía Humana. Madrid, Imprenta y Librería de Nicolas Moya, 1906.

Prives, M.; Lisenkov, N. \& Bushkovich, V. Anatomía Humana. Moscú, MIR, 1984

Rambaud, A. \& Renault, Ch. Origine et développement des os. Paris, F. Chamerot Libraire - Éditeur, 1864.

Romero, R. Anatomical classification of sutural bones. MOJ Anat. Physiol., 3(4):130-1, 2017.

Rouvière, H. \& Delmas, A. Anatomía Humana Descriptiva, Topográfica y Funcional. 10 a ed. Barcelona, Masson, 1999.

Silva, J. \& Araya, C. Umbilicated sutural bones. Int. J. Morphol., 34(2):6169, 2016.

Strambio, G. Elementi di Anatomia Umana Descrittiva, Topografica e Generale. Milan, Francesco Vallardi, 1854.

Testut, L.; Latarjet, A. \& Latarjet, M. Tratado de Anatomía Humana. $9^{\mathrm{a}}$ ed. Barcelona, Salvat, 1965.

Williams, P; Bannister, L; Berry, M.; Collins, P.; Dyson, M.; Dussek, J. \& Ferguson, M. Anatomía de Gray. 38 ed. Madrid, Harcourt Brace, 1998.
Dirección para correspondencia:

Prof. Dr. Juan Luis Silva Rubilar

Académico - Investigador

Facultad de Medicina y Ciencia

Universidad San Sebastián

Campus Los Leones de Providencia

Dirección Lota 2465, Edificio F

Santiago

CHILE

E-mail: juan.silva@uss.cl

Recibido : 30-12-2020

Aceptado: 25-01-2021 